Grand Valley State University

ScholarWorks@GVSU

$9-2005$

\title{
Pathway Curvature and Border Visibility as Predictors of Preference and Danger in Forest Settings
}

Thomas R. Herzog

Grand Valley State University, herzogt@gvsu.edu

Kristin M. Kirk

Grand Valley State University

Follow this and additional works at: https://scholarworks.gvsu.edu/psy_articles

Part of the Psychology Commons

\section{ScholarWorks Citation}

Herzog, Thomas R. and Kirk, Kristin M., "Pathway Curvature and Border Visibility as Predictors of Preference and Danger in Forest Settings" (2005). Peer Reviewed Articles. 25.

https://scholarworks.gvsu.edu/psy_articles/25

This Article is brought to you for free and open access by the Psychology Department at ScholarWorks@GVSU. It has been accepted for inclusion in Peer Reviewed Articles by an authorized administrator of ScholarWorks@GVSU.

For more information, please contact scholarworks@gvsu.edu. 


\section{PATHWAY CURVATURE AND BORDER VISIBILITY AS PREDICTORS OF PREFERENCE AND DANGER IN FOREST SETTINGS}

THOMAS R. HERZOG is a professor of psychology at Grand Valley State University in Allendale, Michigan. His current research focuses on environmental preferences, restorative environments, and the psychology of humor.

KRISTIN M. KIRK is an undergraduate student majoring in psychology at Grand Valley State University.

ABSTRACT: The authors investigated pathway curvature and pathway border visibility as predictors of preference and perceived danger in forest settings. Participants rated 56 forest settings containing pathways for one of four variables-mystery, visual access, pathway length, and pathway width — or for one of four additional predictors. Pathway curvature was unrelated to preference, danger, or mystery. Border visibility and visual access were positively related to preference, negatively related to danger, and positively related to each other. Preference and danger were negatively related. Danger was more strongly related to the predictors than was preference. Mystery had a modest negative relation with preference and substantial relations with danger (positive) and visual access (negative). Factor analysis of the predictor intercorrelations revealed a visibility construct. Visibility scores were positively related to preference and negatively related to danger. For within-forest settings, danger seems to be more salient than preference, and visibility is a major concern.

Keywords: preference; danger; mystery; curvature; visibility

The main purpose of the study reported in this article was to investigate the roles of perceived pathway curvature and perceived pathway border visibility as predictors of preference and danger in forest settings. From a theoretical

AUTHORS' NOTE: Correspondence concerning this article should be addressed to Thomas R. Herzog, Department of Psychology, Grand Valley State University, Allendale, MI 49401; e-mail: herzogt@gvsu.edu.

ENVIRONMENT AND BEHAVIOR, Vol. 37 No. 5, September 2005 620-639

DOI: $10.1177 / 0013916505275306$

(C) 2005 Sage Publications 
standpoint, curvature was of interest because it is one of the setting features traditionally invoked as a component of mystery, one of the cognitive predictors of preference in the Kaplans' informational model of environmental preference (R. Kaplan \& Kaplan, 1989). Mystery refers to the promise that more could be seen if one entered more deeply into a setting. In general, mystery enhances preference, as documented by 30 years of research (for a review, see R. Kaplan, Kaplan, \& Ryan, 1998), although not everyone is convinced (Stamps, 2004). As to the role of pathway curvature, "Some classic examples [of mystery] include the bend in the path and a brightly lit area that is partially obscured by foreground vegetation" (R. Kaplan \& Kaplan, 1989, p. 56). Despite its classic status in theory, pathway curvature has not performed consistently in the few empirical studies in which it was included. Herzog and Miller (1998), investigating urban alleys and field or forest settings containing pathways, found that after controlling for all other predictors in their study via regression analysis, curvature was a positive predictor of mystery, and mystery was a positive predictor of preference. On the other hand, Herzog and Flynn-Smith (2001), in a study of urban alleys, found that whereas mystery was a positive predictor of preference, curvature was a negative predictor, again after controlling for all other predictors in the study. None of the simple correlations relating curvature to either mystery or preference was significant in either of these studies.

The inconsistent performance of pathway curvature might be due to any of several possible causes. First, the variable actually investigated has been perceived curvature, and that variable is clearly confounded with perceived pathway length. It is harder to detect curvature at the end of a long pathway, and thus perceived pathway curvature and pathway length tend to be negatively correlated $(r=-.36$ in Herzog \& Flynn-Smith, 2001). That was the rationale for including perceived length as a control variable in the study by Herzog and Flynn-Smith (2001). Unfortunately, their results failed to clarify the role of curvature as a predictor of preference. A second possibility is that curvature has a nonlinear relation to mystery and thus, indirectly, also to preference. Extreme curvature would disrupt the continuity of information that is central to the concept of mystery. Thus, sharp curvature should be as lacking in mystery as no curvature, and the greatest mystery should occur at intermediate levels of curvature. A fair test of the role of pathway curvature will need to check for the presence of an inverted-U relation between curvature and mystery.

A third possible reason for the inconsistency in previous results for pathway curvature is that although curvature might not be a consistent predictor on its own, it might work together with another predictor in a way that makes sense. It seemed to us that the visual permeability of the pathway border 
might be a good candidate for such a role. Border permeability might be expected to contribute to mystery in much the same way as pathway curvature, with the greatest enhancement in mystery at intermediate levels of permeability. The reasoning is similar: There is little promise of further information at either very high or very low levels of pathway border permeability. Moreover, border permeability might plausibly be expected to interact with pathway curvature in affecting mystery. The relation between either predictor and mystery may vary in magnitude at different levels of the other predictor. As one possible example of such an interaction, an inverted-U relation between pathway curvature and mystery might be reduced in magnitude at either high or low values of border permeability.

To test these possibilities, we included a brand new predictor variable called border visibility, defined as how easy it is "to see through the material that borders the pathway so that you can see what is going on beyond the pathway borders." We checked for both linear and U-shaped relations between pathway curvature and border permeability as predictors and mystery and preference as target variables. In addition, we also explored how curvature and permeability worked together in predicting mystery in a model that included both linear and quadratic terms for each predictor as well as interaction terms up to the level of quadratic by quadratic. A final possibility is that pathway curvature really has no consistent relation to either mystery or preference. This would imply that although a bend in the path can be associated with high mystery or preference, it can also be associated with low mystery or preference, and the same can be said for a straight pathway. If so, the next task would be to determine the circumstances in which either a curved or a straight path would enhance mystery or preference.

Thus far we have considered only preference as a dependent variable, but there are excellent reasons for looking at perceived danger as well. Although one might expect a negative relation between preference and danger, an expectation confirmed in several studies (see Herzog \& Kropscott, 2004, for a review), danger has some advantages over preference as a dependent variable. Theoretically, one might expect danger to be more salient than preference for humans. On evolutionary grounds, preference is less urgent in that it can be deferred, but generally danger must be given a high priority or survival is threatened. Given the difference in salience, the pattern of predictability might be better for the more salient variable, danger. Is there any empirical support for these speculations? In a series of studies, Herzog and colleagues have provided some evidence supporting the proposition that preference and danger are not simply polar opposites (Herzog \& Flynn-Smith, 2001; Herzog \& Kropscott, 2004; Herzog \& Kutzli, 2002; Herzog \& Miller, 1998). They have found a tendency for mystery to be positively related to both preference 
and danger, especially after controlling for other predictor variables. Specifically, Herzog and Kropscott (2004) found that a significant negative simple correlation between mystery and preference $(r=-.39)$ became a significant positive partial correlation $(r=.34)$ with visual access (described below) as the partialed variable. Mystery was positively correlated with danger before and after partialing. On the question of differential predictability, Table 1 is informative. In each of five previous studies that have included both preference and danger, the mean absolute value of the correlation of all predictor variables with danger was higher than it was with preference. The unweighted average of the correlations across all five studies is . 32 for preference and .55 for danger. Thus, we included perceived danger in the present study and expected, with considerable confidence, that it would have a stronger relation to the predictor variables than would preference.

Including pathway border visibility as a predictor variable raises the general issue of visibility. On theoretical grounds, one might expect that visibility, the ability to see unhindered into a setting, generally would be a desirable property in a forest setting. Blocked views make it difficult to comprehend any setting and discourage exploration. Thus, they have been associated both theoretically (S. Kaplan, 1979) and empirically (e.g., Herzog, 1992) with depressed preference. Conversely, visual access has been associated with positive reactions to forest settings (e.g., Rudell, Gramann, Rudis, \& Westphal, 1989). Likewise, the burgeoning literature on fear of crime highlights the fact that blocked views afford concealment for criminals and thus are associated with danger reactions (e.g., Fisher \& Nasar, 1992; Loewen, Steel, \& Suedfeld, 1993; Nasar \& Fisher, 1993; Nasar \& Jones, 1997; Schroeder \& Anderson, 1984; Shaffer \& Anderson, 1983). Two recent studies of preference and danger in natural settings (Herzog \& Kropscott, 2004; Herzog \& Kutzli, 2002) have included a predictor variable called visual access, defined as the ability to see all parts of a setting without having one's view blocked or interfered with. In both studies visual access was a significant predictor of preference (a positive relation) and an even stronger predictor of danger (a negative relation). We also included visual access in the present study and expected a similar pattern of results. Furthermore, by including visual access, we were able to compare the simple correlation between mystery and preference with their partial correlation, controlling for visual access, as described in the previous paragraph. In addition, because it seems likely that pathway border visibility is one component of visual access, we expected a positive correlation between the two variables. Likewise, it would seem that longer and wider pathways should afford greater visual access. Thus, we also expected positive relations among visual access and both pathway length and pathway width. 


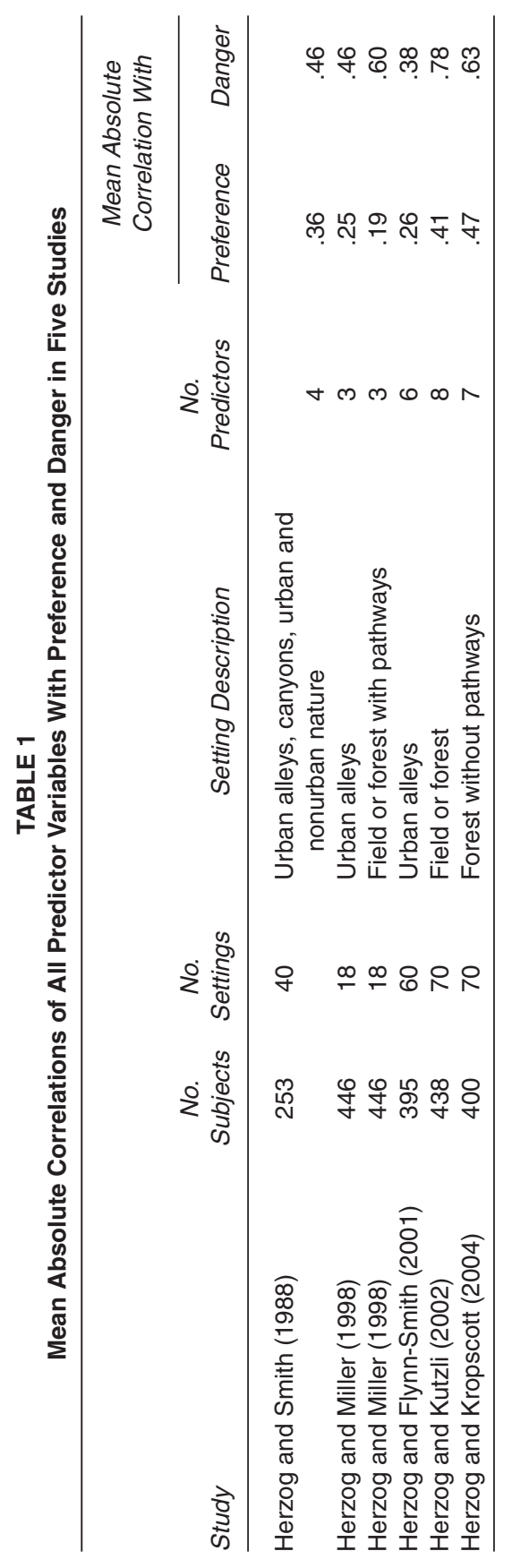


Finally, we had an opportunity to test a suggestion made by Herzog and Kropscott (2004). As noted earlier, they found a negative correlation ( $r=$ -.39 ) between mystery and preference in their sample of forest settings without pathways. There was also a very strong negative correlation between mystery and visual access $(r=-.92)$, suggesting that mystery was largely a matter of reduced visual access. They speculated that such a pattern of relations might be peculiar to within-forest settings that lacked pathways and proposed a comparison of such settings with forest settings that have pathways. Because our sample consisted of forest settings with pathways, we were able to assess their implied prediction that a more typical pattern of results (positive relations among the three variables) would occur with such settings.

In summary, we investigated preference and danger reactions to a sample of within-forest settings containing pathways. Our primary interest was in two predictors: pathway curvature and pathway border visibility. We also included two other predictors primarily as controls: pathway length and pathway width. We regard all four of these predictors as involving relatively immediate perceptual properties. At a more inferential level, we also included the predictors mystery and visual access. Theoretical considerations suggest that pathway curvature might have an inverted-U relation to mystery. On intuitive grounds, we expected that pathway length, width, and (especially) border visibility would contribute positively to visual access. Given this set of predictors, we were able to assess how pathway curvature and border visibility worked together in contributing to mystery as well as in accounting for preference and danger reactions. We could also assess a number of other predictions reviewed above involving (a) the relation between preference and danger; (b) the differential predictability of preference and danger; (c) relations among the inferential predictors, mystery and visual access, and the target variables, preference and danger; and (d) relations among the perceptual predictors (pathway curvature, length, width, and border visibility) and the inferential predictors.

\section{METHOD}

\section{PARTICIPANTS}

The sample of raters consisted of 563 undergraduate students (153 men, 410 women) at a university in the midwestern United States. Participation fulfilled a course requirement for introductory psychology. A total of 22 

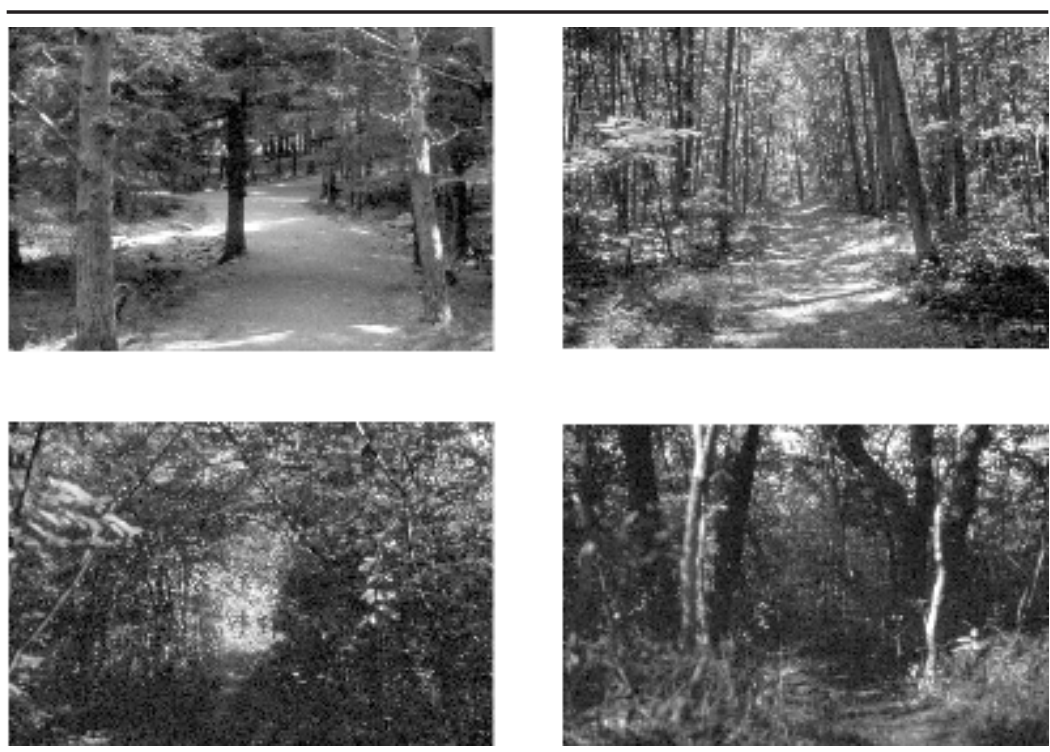

Figure 1: Examples of Settings Rated High in Preference and Low in Danger (Top Row) or Low in Preference and High in Danger (Bottom Row)

NOTE: Setting scores for these settings are presented in Table 3.

sessions were conducted, with the number of participants per session ranging from 13 to 36 .

\section{STIMULI}

The settings consisted of 56 color slides of within-forest environments containing pathways. This was a purposive sample in several respects. Each setting had to be a within-forest view, and it had to contain a pathway. In addition, we attempted to sample broadly with respect to each of the four perceptual predictors: pathway curvature, length, width, and border visibility. We also attempted to include all combinations of high and low values of curvature, length, and visibility. Figures 1 and 2 provide some examples of the settings. No settings contained people. All were photographed in spring, summer, or early fall. All slides were oriented horizontally.

\section{PROCEDURE}

All participants in each session rated each of the 56 settings on only one of the eight measured variables. All ratings used a 5-point scale ranging from $\mathrm{A}$ 

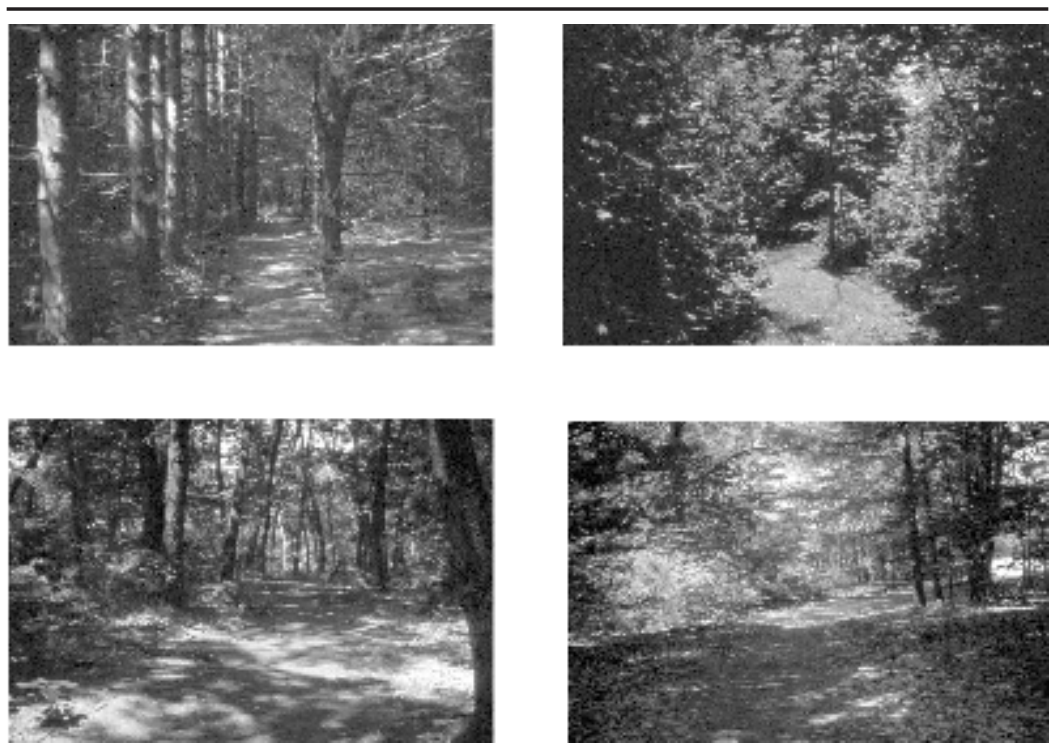

Figure 2: Examples of Settings Rated High (Top Row) or Low (Bottom Row) in Mystery

NOTE: Setting scores for these settings are presented in Table 3.

(very high) to $\mathrm{E}$ (not at all). The letters A through $\mathrm{E}$ were later converted to the numbers 5 through 1 , respectively, for analysis. The two target variables were preference and danger. Preference was defined as

How much do you like the setting? This is your own personal degree of liking for the setting as a setting, NOT as a picture. You don't have to worry about whether you're right or wrong or whether you agree with anybody else.

Danger was defined as "How dangerous is this setting? How likely is it that you could be harmed in this setting?" The four perceptual predictors were pathway curvature, length, width, and border visibility. Pathway curvature was defined as

Consider how much the pathway curves or bends. The strongest curvature you will see will be about 90 degrees or a right-angle bend. Such settings should be rated high (A) in Curvature. If the pathway is completely straight, with no curvature at all, rate the setting low (E) in Curvature.

Pathway length was defined as "How long does the pathway appear to be from its nearest to its farthest visible point?" Likewise, pathway width was 
defined as "How wide does the pathway appear to be from its left-hand border or side to its right-hand border or side?" Pathway border visibility was defined as "How easy is it to see through the material that borders the pathway so that you can see what is going on beyond the pathway borders?" The two intervening variables assessed were mystery and visual access. Mystery was defined as "How much does the setting promise more to be seen if you could walk deeper into it? Does the setting seem to invite you to enter more deeply into it and thereby learn more?" Visual access was defined as "How easy is it to see into this setting? How well can you see all parts of this setting without having your view blocked or interfered with?"

Sessions proceeded as follows. After explaining the task and obtaining informed consent, the first 10 slides were shown briefly ( 5 seconds per slide) without being rated to familiarize participants with the range of settings to be encountered. Then participants rated 60 slides, presented in two sets of 30 slides each, with a 2-minute rest between sets. Viewing time was 15 seconds per slide in all sessions. The first and last slides within each set were fillers, intended to absorb any beginning- or end-of-set effects. The remaining 56 slides yielded the data for analysis. These slides were presented in one of two orders. The first order was used for the first 11 sessions, the second order was used for the last 11 sessions. Within each block of sessions using a given slide order, there were 4 sessions devoted to preference and 1 session devoted to each of the other seven rated variables. ${ }^{1}$ Aside from the constraints on the ordering of sessions just noted, the ordering of sessions was randomized. One of the slide presentation orders was generated randomly, and the second presentation order was derived by interchanging the halves of the first order. Final sample sizes were 215 for preference, 56 for danger, 54 for pathway length, 52 for pathway border visibility, 51 for mystery, 50 for pathway width, 44 for visual access, and 41 for pathway curvature.

\section{RESULTS}

All analyses were based on setting scores as raw scores. A setting score is the mean score for each setting based on all participants who completed one of the rating tasks. Thus, for each rated variable, every setting had a setting score, and settings typically were the units of analysis. Internal-consistency reliability coefficients (Cronbach's $\alpha$ ), based on settings as cases and participants as items, ranged from .90 for mystery to .99 for pathway width.

Table 2 presents descriptive statistics for the eight rated variables. Two points are noteworthy. First, the four perceptual predictors had the widest 
range of score values, all spanning at least 3 scale units and indicating that we were successful in broadly sampling these variables. Second, overall mean scores tended to fall near the midpoint of the 5-point scale, with the notable exception of danger, which generally had low ratings $(M=2.24)$. This is typical of the way danger is rated in forest settings (e.g., Herzog \& Kropscott, 2004).

Figure 1 provides some useful imagery. It shows examples of settings rated at the extremes of preference and danger. The top four rows of Table 3 contain the setting scores on all eight rated variables for the four settings in Figure 1. It is immediately apparent that high preference was paired with low danger and vice versa. It is also clear that the high preference, low danger settings tended to be higher in visual access as well as pathway length, width, and border visibility than the low preference, high danger settings.

Table 4 presents the correlations among the rated variables based on all 56 settings. Given the number of correlations, we accord the greatest weight to those that are significant at $p<.001$. These results bear on several of the hypotheses proposed in the introduction. First, preference and danger were negatively correlated, confirming our prediction. Second, danger was more strongly correlated with all of the predictor variables $(M|r|=.52)$ than was preference $(M|r|=.38)$, also confirming our prediction. Third, mystery had a strong positive correlation with danger, but its weak correlation with preference $(p<.05)$ was negative, continuing a recent trend toward negative correlations between mystery and preference (e.g., Herzog \& Kropscott, 2004; Herzog \& Kutzli, 2002). In addition, mystery was negatively correlated with visual access, the same relation reported by Herzog and Kropscott (2004) for forest settings without pathways. In general, the predictor variables tended to have opposite-sign correlations with preference and danger. Fourth, visual access was the best predictor of both target variables (a positive predictor of preference and a negative predictor of danger), confirming our prediction. Fifth, pathway curvature was uncorrelated with either target variable and also with mystery. Its only significant relation was a negative correlation with pathway length, confirming our prior intuition. Pathway border visibility, on the other hand, was a strong predictor of both target variables, with the same pattern of correlations as visual access. Sixth, the other three perceptual predictors (pathway length, width, and border visibility) were positively correlated with visual access, as predicted. Finally, mystery, visual access, and border visibility were all intercorrelated (with mystery negatively related to the other two variables), suggesting an underlying visibility factor similar to the one obtained in two previous studies (Herzog \& Kropscott, 2004; Herzog \& Kutzli, 2002). 


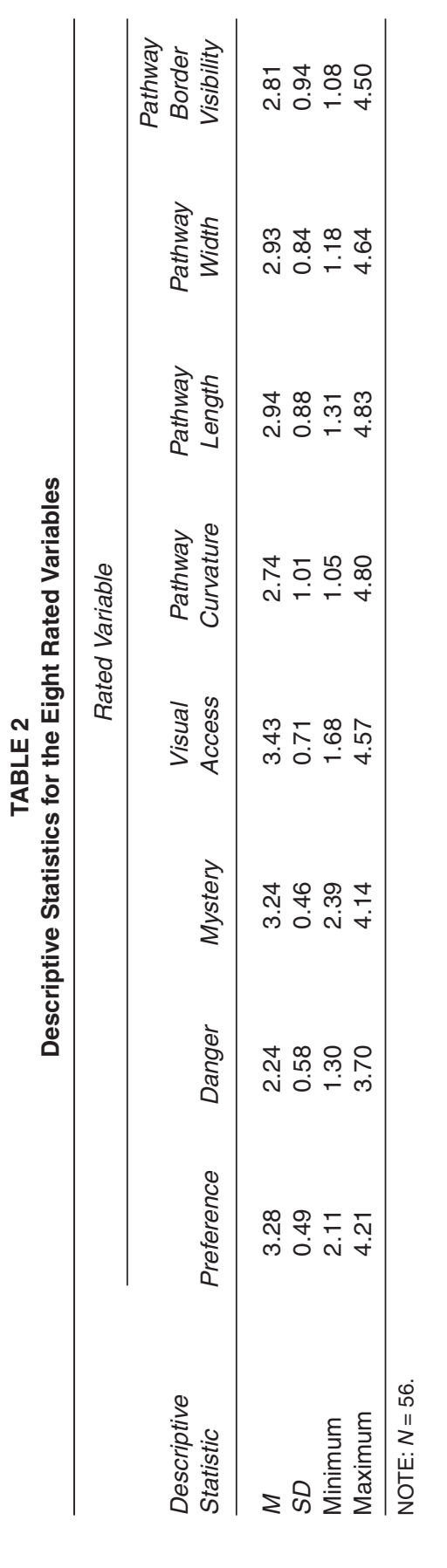




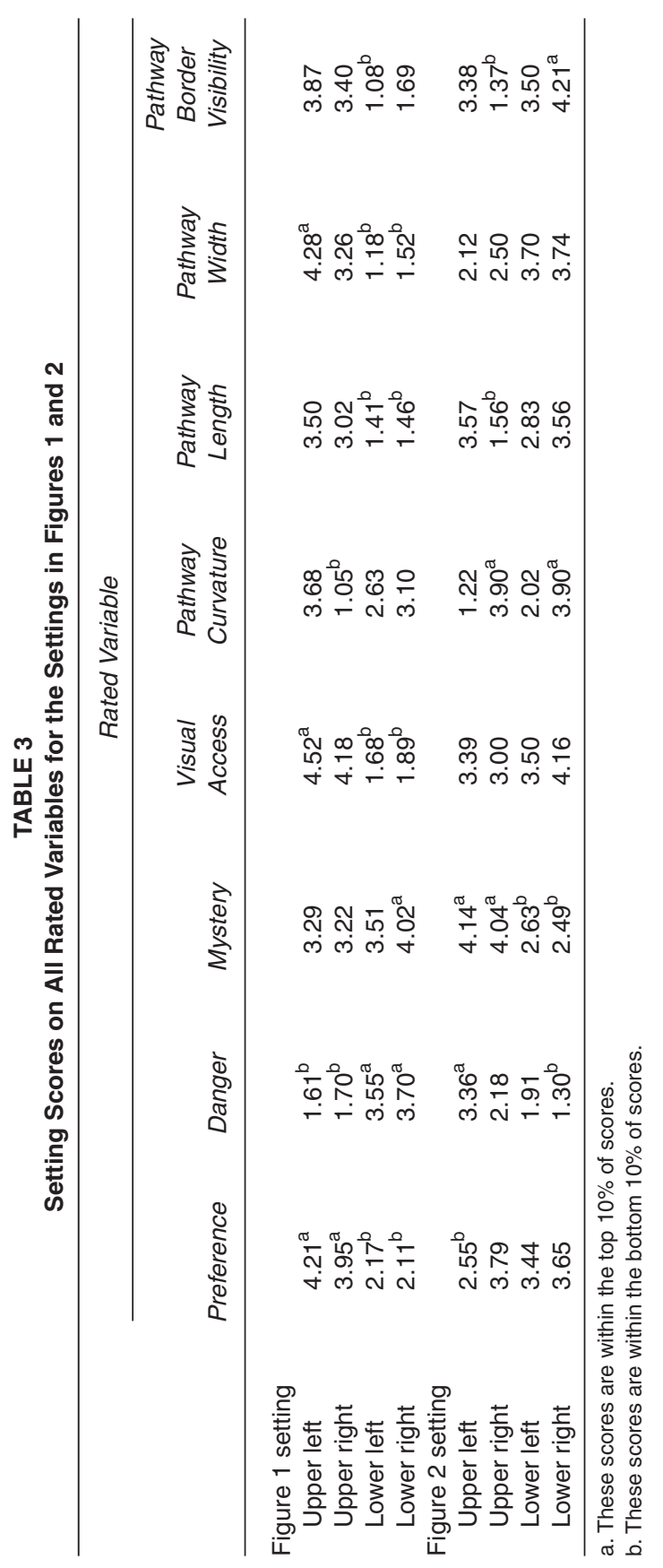


TABLE 4

Correlations Among All Rated Variables for All Settings

\begin{tabular}{|c|c|c|c|c|c|c|c|c|}
\hline & 1 & 2 & 3 & 4 & 5 & 6 & 7 & 8 \\
\hline 1. Preference & - & & & & & & & \\
\hline 2. Danger & $-.76^{\star *}$ & - & & & & & & \\
\hline $\begin{array}{l}\text { 3. Mystery } \\
\text { 4. Visual }\end{array}$ & -.29 & $.61^{* *}$ & - & & & & & \\
\hline $\begin{array}{c}\text { access } \\
\text { 5. Pathway }\end{array}$ & $.72^{\star *}$ & $-.85^{\star *}$ & $-.49^{\star *}$ & - & & & & \\
\hline $\begin{array}{l}\text { curvature } \\
\text { 6. Pathway }\end{array}$ & -.06 & -.04 & -.18 & -.12 & - & & & \\
\hline $\begin{array}{l}\text { length } \\
\text { 7. Pathway }\end{array}$ & .31 & $-.40^{\star}$ & -.21 & $.56^{\star \star}$ & $-.59^{\star \star}$ & - & & \\
\hline $\begin{array}{l}\text { width } \\
\text { 8. Pathway } \\
\text { border }\end{array}$ & .33 & $-.62^{\star \star}$ & $-.43^{*}$ & $.55^{\star \star}$ & .22 & .21 & - & \\
\hline visibility & $.57^{\star *}$ & $-.57^{\star *}$ & $-.67^{* *}$ & $.64^{* *}$ & .02 & $.38^{*}$ & .32 & 一 \\
\hline
\end{tabular}

NOTE: $N=56$

${ }^{*} p<.01 .{ }^{* *} p<.001$.

Further evaluation of some of our hypotheses required that we examine partial relations, nonlinear relations, and interactions. To assess the relation between mystery and preference while controlling for visual access, we computed the partial correlation between mystery and preference with visual access as the partialed variable. That correlation was $.11(p>.05)$. The sign of the partial correlation matches the results of Herzog and Kropscott (2004), but the magnitude falls short. Meanwhile, the partial correlation between mystery and danger, with visual access partialed out, was .42 $(p<.005)$, as compared to .61 for the simple correlation (Table 4 ). To assess the possibility of an inverted-U relation between pathway curvature or border visibility as predictors and mystery or preference as target variables, we ran quadratic regressions separately for each target variable and each predictor. ${ }^{2}$ The quadratic component was significant only in the analysis with preference as the target variable and pathway curvature as the predictor $(p=.014)$. However, the quadratic regression coefficient was positive (.17), indicating an upright$\mathrm{U}$ relation, contrary to our prediction. We also ran a regression analysis with mystery as the target variable and both pathway curvature and border visibility as predictors. The model included both linear and quadratic components for each predictor as well as all interaction terms up through the level of quadratic by quadratic. We used step-down analysis as advocated by Aiken and West (1991) to simplify the model as much as possible. The result was that all higher-order terms were eliminated, leaving a simple linear model involving 
the two predictors in which only border visibility was significant $(\mathrm{p}<.001)$ as a negative predictor (partial $r=-.67$ ). This is the same pattern as in Table 4, where border visibility has a significant negative correlation with mystery, whereas pathway curvature and mystery are uncorrelated. Given that nonlinear relations involving pathway curvature and border visibility had been ruled out, we ran a final set of analyses to check for linear interaction between the two predictors while controlling for the other two perceptual predictors. We did separate regression analyses with preference, mystery, and danger as target variables. Each analysis included all four of the perceptual variables (pathway length, width, curvature, and border visibility) as predictors as well as a cross-product term representing the interaction between curvature and border visibility. The important finding was that the interaction of curvature and border visibility was not significant in any of these analyses. When we ran the regressions without the interaction term, the results generally confirmed the findings of Table 4: Border visibility was a strong predictor in all three regressions (positive for preference, negative for danger and mystery; $p<.002$ in all cases), and the only other significant predictor was a negative partial relation between pathway width and danger. ${ }^{3}$

As noted earlier, Table 4 suggests the presence of an underlying visibility factor composed of (at least) visual access, border visibility, and mystery. To check this, we factor analyzed the intercorrelations of the six predictor variables (principal-axis factoring, varimax rotation). Table 5 presents the factor loading for the two factors with eigenvalues greater than 1 . The two factors accounted for $38 \%$ and $25 \%$ of the variance in the data, respectively, after rotation. After factor extraction, communalities ranged from .35 for pathway width to .77 for pathway curvature. As we suspected, the first factor seems to be about visibility, with positive loadings for visual access and border visibility and a negative loading for mystery. As was the case for both Herzog and Kropscott (2004) and Herzog and Kutzli (2002), mystery has a negative loading on the visibility factor. Pathway width also makes a modest positive contribution to visibility. The second factor is pretty much a matter of pathway curvature (positive loading) and length (negative loading). Thus, the highest standing on this factor goes to settings with short and sharply curved pathways, the lowest standings to settings with long, straight pathways. Given the negative correlation between pathway curvature and length, we can roughly think of this factor as perceived pathway curvature. The correlation between factor scores on this factor and setting scores for pathway curvature was .93.

Our final analyses assessed these derived predictor constructs, visibility and curvature, and the target variables, preference and danger. To do so, we obtained factor scores for each of the two factors using the regression approach. The correlation between the factor scores for visibility and 
TABLE 5

Factor Loadings From the Factor Analysis of the Six Rated Predictor Variables

\begin{tabular}{lrr}
\hline & \multicolumn{2}{c}{ Factors } \\
\cline { 2 - 3 } Variables & $I$ & \multicolumn{1}{c}{ II } \\
\hline Mystery & -.75 & \\
Visual access & .79 & .32 \\
Pathway curvature & .20 & .86 \\
Pathway length & .38 & -.78 \\
Pathway width & .59 & \\
Pathway border visibility & .76 & -.13 \\
\hline
\end{tabular}

NOTE: Only loadings $>|.10|$ are shown. Factors I and II were interpreted as visibility and perceived pathway curvature, respectively.

curvature was -.03 . Visibility was positively correlated with preference and negatively correlated with danger $(r=.61$ and -.83 , respectively; $p<.001$ in both cases). Curvature was not significantly correlated with either target variable ( $r=-.20$ for preference and .16 for danger; $p>.05$ in both cases). Regression analyses including both predictors yielded the same pattern: visibility a significant positive predictor of preference and a significant negative predictor of danger $(p<.001$ in both cases) and curvature not a significant predictor of either target variable. We also checked to see if the predictors interacted. They did not for either target variable.

\section{DISCUSSION}

We begin by acknowledging potential limitations of our study. Two perennial concerns are the population of raters and the medium of presentationcolor slides. We concede that results for predominantly female college students might not generalize to other age and gender groups (Balling \& Falk, 1982; Herzog, Herbert, Kaplan, \& Crooks, 2000; Zube, Pitt, \& Evans, 1983). Although concerns about using color slides as a medium of presentation have been expressed (e.g., Heft \& Nasar, 2000; Scott \& Canter, 1997), the validity of the medium for aggregate results and static visual attributes of environments is supported (e.g., Hershberger \& Cass, 1973; Hull \& Stewart, 1992; Zube, Simcox, \& Law, 1987). The setting domain, within-forest settings, may impose further restrictions on generalization. For certain variables, there may also be questions about valid measurement. Concerns about setting domain and measurement validity are incorporated into the discussion below. 
Within the limitations above, what have we learned? Checking the hypotheses and hunches outlined in the introduction, we conclude that perceived pathway curvature has little to do with preference, danger, or mystery. It was not an effective predictor in our analyses, simply or partially, and it did not interact with border visibility. One could always question the measurement validity of pathway curvature and propose that objective measures of actual curvature might still be effective. Research containing such a measure would be necessary to settle the matter. Pending such results, we confess to skepticism about this proposal because we are committed to the proposition that it is perception that matters, even when it differs from reality. As regards mystery and assuming for the moment the validity of the mystery ratings (more on that below), the results seem to be telling us that perceived curvature is not related in any simple way to the impression of mystery. This is not to say that curvature cannot enhance mystery but only that a straight pathway can be just as mysterious as a curved one. Thus, as Table 3 indicates, the two settings in the upper row of Figure 2 were seen as equally high in mystery even though they differed substantially in perceived pathway curvature. Likewise, the two settings in the bottom row of Figure 2 were seen as equally low in mystery even though they also differed substantially in perceived curvature. On this interpretation, curvature can play a role, but other factors can override it.

Meanwhile, we can also conclude that pathway border visibility was an effective predictor, presumably as one component of overall visibility, the one underlying construct in our set of predictors that was associated with the target variables. This is now the third study, following Herzog and Kutzli (2002) and Herzog and Kropscott (2004), in which mystery and visual access were subsumed under a visibility factor, mystery as a negative contributor. In all of these studies, visibility was a strong positive predictor of preference and an even stronger negative predictor of danger. It is important to emphasize that this pattern of relations may be limited to settings with low to moderate visibility such as within-forest settings. Schroeder and Anderson (1984) found a negative correlation between view distance and scenic preference in a sample of settings that included within-forest views as well as open, treeless, park landscapes. (It is worth noting, however, that even with their broader range of settings, Schroeder and Anderson found the same kind of negative relation between visibility and perceived danger that we did.) It seems reasonable to conclude that visibility is a paramount concern in evaluative reactions, especially fear-of-danger reactions, to environmental settings with limited visibility. This conclusion has practical implications. A useful guideline would be that when including nature or mystery in settings with limited visibility, take steps to enhance visibility. Suggestions for 
achieving this goal can be found in Herzog and Kutzli (2002), Herzog and Leverich (2003), Herzog and Miller (1998), Kuo, Bacaicoa, and Sullivan (1998), and Nasar and Jones (1997).

Specifically regarding danger as a target variable, this study adds to the accumulating evidence (reviewed in the introduction) for two conclusions. First, preference and danger are negatively correlated. Computed within setting domains and averaged across five studies including this one (Herzog \& Flynn-Smith, 2001; Herzog \& Kropscott, 2004; Herzog \& Kutzli, 2002; Herzog \& Miller, 1998) for which data are available, the average correlation between the two variables is -.53 . Thus, although substantially correlated, preference and danger should be viewed as distinct constructs. As noted in the introduction, mystery has tended to correlate positively with both preference and danger in recent studies, after controlling for other predictors, especially those related to visibility. In this study, after controlling for visual access, the partial correlations of mystery with preference and danger were .11 and .42 , respectively. The trend toward positive partial correlations with both target variables continues, but the correlation with preference was not significant. The second conclusion regarding danger is that it seems to be more salient than preference as a target variable in that it has stronger correlations with predictor variables. Confirming our prediction and continuing the trend of recent studies reviewed in the introduction, in this study the mean absolute value of all correlations involving predictor variables with the two target variables was .52 for danger and .38 for preference. Given that a heightened sensitivity to danger makes good sense from an evolutionary perspective, it would be of interest to devise further means of testing this hypothesis.

We return finally to mystery, which has shown a tendency recently to produce negative correlations, though not always significant, with both preference and visual access (which are positively correlated with each other; Herzog \& Kropscott, 2004; Herzog \& Kutzli, 2002; this study). Herzog and Kropscott (2004) suggested that the problem might be confined to withinforest settings without pathways, but the results of this study decisively contradict that proposal. It is still possible and worth investigating that inclusion of settings with even greater openness, such as fields, would produce the accustomed positive correlation between mystery and preference. But what should we conclude regarding within-forest settings? Three possibilities come to mind. First, the negative correlations between mystery and preference are a matter of chance, and the trend will not continue. Second, perhaps our measure of mystery may be malfunctioning in the context of within-forest settings with limited visibility. Returning to Figure 2, why is the upper-left setting ranked high in mystery? It cannot be because of enhanced visual 
access associated with the relatively long, straight pathway. The visual access score for that setting (3.39, Table 3) ranks 29.5 out of 56 . If there is something else about the setting that draws one into it thereby accounting for the mystery score, it is not a form of mystery that enhances preference because this setting ranks among the lowest in preference. Even more problematic is the lower-right setting of Figure 2. It ranked among the lowest in mystery even though it has two classic indicators of mystery, the curving pathway and the partially obscured opening into the clearing at the end of the pathway. These examples suggest that our raters may not have adequately understood the concept of mystery and instead defaulted largely to lack of visual access when rating mystery. They may have confused mystery with surprise, a classic confusion first described by S. Kaplan and Kaplan (1982).

A third possible explanation for the negative correlations between mystery and preference is that our definition of mystery is fine and that we have simply discovered a type of setting in which mystery is negatively related to preference. The most important characteristic of this setting type would appear to be a predominance of dense vegetation or other material that limits visibility. Densely vegetated forest settings clearly fit this specification. In such settings, mystery may truly function atypically. It may be incorporated into a visibility construct, as a negative indicator, with predictable results. After controlling for visibility, the small remaining systematic variability in mystery tends toward a positive relation with preference. This view suggests that there are isolated setting categories that violate the general prediction from the Kaplans' (R. Kaplan \& Kaplan, 1989) informational model that mystery enhances preference. Such exceptional cases would imply boundary conditions on the generality of the model. Given the scarcity of such cases so far discovered, they would also seem to be exceptions that prove the general rule. To shed light on the possibilities discussed here, further investigation of setting domains, especially those differing substantially in visibility or vegetation density, and rating methods, especially those differing in the amount of instruction in the meaning of the mystery construct, may prove helpful.

Our major conclusions are that danger seems to be more salient than preference and that visibility is a potent predictor of both reactions for within-forest settings. Visibility includes such features as pathway length, width, and border permeability as well as visual access to the rest of the setting. Although visibility was our best predictor, we do not endorse a singlepredictor approach in accounting for either preference or danger. Only one predictor from the Kaplans' (R. Kaplan \& Kaplan, 1989) informational model, mystery, was represented in this study. Their other predictors (complexity, coherence, legibility) are also surely relevant and unlikely to be subsumed by the visibility construct. With less assurance, we suggest that 
perceived pathway curvature has little relation to danger or preference, although it should on theoretical grounds. Finally, we note that findings of modest negative correlations between mystery and preference pose a challenge to current thinking about mystery. Resolving that challenge may shed valuable light on how mystery functions in certain contexts, thereby furthering our understanding of the construct.

\section{NOTES}

1. The extra sessions for preference afforded us the option of factor analyzing the preference ratings, but we ultimately decided not to do so. Herzog and Kropscott (2004) factor analyzed the preference ratings for their within-forest settings and found that the factor-based setting categories were highly redundant with their visual-access-rated predictor $(r=.89)$.

2. We did not check for nonlinear relations involving danger because there was no compelling theoretical reason to do so.

3. We checked for multicollinearity problems in all of our regression analyses. There were none.

\section{REFERENCES}

Aiken, L. S., \& West, S. G. (1991). Multiple regression: Testing and interpreting interactions. Newbury Park, CA: Sage.

Balling, J. D., \& Falk, J. H. (1982). Development of visual preference for natural environments. Environment and Behavior, 14, 5-28.

Fisher, B. S., \& Nasar, J. L. (1992). Fear of crime in relation to three exterior site features: Prospect, refuge, and escape. Environment and Behavior, 24, 35-65.

Heft, H., \& Nasar, J. L. (2000). Evaluating environmental scenes using dynamic versus static displays. Environment and Behavior, 32, 301-322.

Hershberger, R. G., \& Cass, R. C. (1973). The adequacy of various media as representations of the designed environment. Man-Environment Systems, 3, 371-372.

Herzog, T. R. (1992). A cognitive analysis of preference for urban spaces. Journal of Environmental Psychology, 12, 237-248.

Herzog, T. R., \& Flynn-Smith, J. A. (2001). Preference and perceived danger as a function of the perceived curvature, length, and width of urban alleys. Environment and Behavior, 33, 655668.

Herzog, T. R., Herbert, E. J., Kaplan, R., \& Crooks, C. L. (2000). Cultural and developmental comparisons of landscape perceptions and preferences. Environment and Behavior, 32, 323 346.

Herzog, T. R., \& Kropscott, L. S. (2004). Legibility, mystery, and visual access as predictors of preference and perceived danger in forest settings without pathways. Environment and Behavior, 36, 659-677. 
Herzog, T. R., \& Kutzli, G. E. (2002). Preference and perceived danger in field/forest settings. Environment and Behavior, 34, 858-874.

Herzog, T. R., \& Leverich, O. L. (2003). Searching for legibility. Environment and Behavior, 35, 459-477.

Herzog, T. R., \& Miller, E. J. (1998). The role of mystery in perceived danger and environmental preference. Environment and Behavior, 30, 429-449.

Herzog, T. R., \& Smith, G. A. (1988). Danger, mystery, and environmental preference. Environment and Behavior, 20, 320-344.

Hull, R. B., IV, \& Stewart, W. P. (1992). Validity of photo-based scenic beauty judgments. Journal of Environmental Psychology, 12, 101-114.

Kaplan, R., \& Kaplan, S. (1989). The experience of nature: A psychological perspective. New York: Cambridge University Press.

Kaplan, R., Kaplan, S., \& Ryan, R. L. (1998). With people in mind: Design and management of everyday nature. Washington, DC: Island.

Kaplan, S. (1979). Concerning the power of content-identifying methodologies. In T. C. Daniel \& E. H. Zube (Eds.), Assessing amenity resource values (Tech. Rep. No. RM-68, pp. 4-13). Fort Collins, CO: U.S. Department of Agriculture Forest Service.

Kaplan, S., \& Kaplan, R. (1982). Cognition and environment: Functioning in an uncertain world. New York: Praeger.

Kuo, F. E., Bacaicoa, M., \& Sullivan, W. C. (1998). Transforming inner-city landscapes: Trees, sense of safety, and preference. Environment and Behavior, 30, 28-59.

Loewen, L. J., Steel, G. D., \& Suedfeld, P. (1993). Perceived safety from crime in the urban environment. Journal of Environmental Psychology, 13, 323-331.

Nasar, J. L., \& Fisher, B. (1993). 'Hot spots' of fear and crime: A multi-method investigation. Journal of Environmental Psychology, 13, 187-206.

Nasar, J. L., \& Jones, K. M. (1997). Landscapes of fear and stress. Environment and Behavior, 29, 291-323.

Ruddell, E. J., Gramann, J. H., Rudis, V. A., \& Westphal, J. M. (1989). The psychological utility of visual penetration in near-view forest scenic-beauty models. Environment and Behavior, $21,393-412$.

Schroeder, H. W., \& Anderson, L. M. (1984). Perception of personal safety in urban recreation sites. Journal of Leisure Research, 16, 178-194.

Scott, M. J., \& Canter, D. V. (1997). Picture or place? A multiple sorting study of landscape. Journal of Environmental Psychology, 17, 263-281.

Shaffer, G. S., \& Anderson, L. M. (1983). Perceptions of the security and attractiveness of urban parking lots. Journal of Environmental Psychology, 5, 311-323.

Stamps, A. E., III. (2004). Mystery, complexity, legibility, and coherence: A meta-analysis. Journal of Environmental Psychology, 24, 1-16.

Zube, E. H., Pitt, D. G., \& Evans, G. W. (1983). A lifespan developmental study of landscape assessment. Journal of Environmental Psychology, 3, 115-128.

Zube, E. H., Simcox, D. E., \& Law, C. S. (1987). Perceptual landscape simulations: History and prospect. Landscape Journal, 6, 62-80. 